\title{
Exploration of the Transition from Harrison Type-A Kinetics to Type-B Kinetics Regimes in Grain Boundary Diffusion*
}

\author{
I.V. Belova ${ }^{1, a}$ and G.E. Murch ${ }^{1, b}$ \\ ${ }^{1}$ Diffusion in Solids Group \\ Centre for Geotechnical and Materials Modelling \\ School of Engineering \\ The University of Newcastle \\ Callaghan \\ NSW 2308 \\ Australia \\ a Irina.Belova@newcastle.edu.au, ${ }^{\text {b }}$ Graeme.Murch@newcastle.edu.au
}

Keywords: grain boundary diffusion, effective diffusion, Harrison kinetics regimes

\begin{abstract}
We model the grain boundary tracer diffusion problem by constructing a 3D structure consisting of cubic grains each of equal volume. We build the structure in such a way that no four cubes have a common edge. It is shown that the transition point between Harrison Type-A and Type-B kinetics regimes occurs at a diffusant diffusion length roughly an order of magnitude smaller than for the extensively studied case of parallel grain boundary slabs. For two dimensional squares the transition point occurs at a diffusion length roughly a factor of five smaller than for parallel grain boundary slabs. Thus we can draw the conclusion that dimensionality and geometric shape are both important factors in the parametric analysis of the grain boundary diffusion problem.
\end{abstract}

\section{Introduction}

The Harrison Type-A kinetics regime [1] refers to the situation where the diffusion length of tracer atoms from a tracer source at the surface is substantially greater than the grain size $(L)$. The corresponding tracer concentration depth profile then provides the effective diffusivity $D_{\text {eff. For }}$ parallel grain boundary 'slabs' to the surface (this is a common model in this area), $D_{\text {eff }}$ is then simply given (for self-diffusion) by the Hart Equation [2]:

$$
D_{\text {eff }}=g D_{\mathrm{gb}}+(1-g) D_{1}
$$

where $D_{\mathrm{gb}}$ is the grain boundary diffusivity, $D_{\mathrm{l}}$ is the lattice diffusivity and $g$ is the fraction of sites in the grain boundary. Eq. 1 is exact for grain boundary slabs parallel to the diffusion direction.

Harrison Type-B kinetics regime refers to a situation where the diffusion length of the tracer atoms is less than the grain size. Then the corresponding depth profile has two regions. The first region provides the lattice diffusivity whilst the second or tail region provides (for self-diffusion) the product of the grain boundary diffusivity and the grain boundary width $\delta$.

The transition point between Harrison Type-A kinetics and Harrison Type-B kinetics was originally estimated to occur at $\Lambda\left(=L /\left(D_{l} t\right)^{1 / 2}\right)=0.0033$ for spherical grains [1]. Recently, the transition point from Harrison Type-A to Type-B kinetics regimes was greatly revised by the present authors as a result of extensive detailed Lattice Monte Carlo simulations of tracer depth concentration profiles as a function of the distance between grain boundaries [3]. The model used in that study was the usual parallel grain boundary slab model. In that study, it was shown that the

\footnotetext{
* This paper is dedicated to the memory of the late Prof. Alan Le Claire.
} 
upper limit for Harrison type-A kinetics regime occurs when $\Lambda$ is approximately equal to 0.4 and this is essentially independent of the $\beta$, frequently called the 'Le Claire parameter', $\left(\beta=\delta D_{\mathrm{gb}} / 2 D_{1}\right.$ $\left.\left(D_{1} t\right)^{1 / 2}\right)$. We found a small transition region between 0.4 and 0.2 , which is somewhat dependent on $\beta$ before the Harrison type-B kinetics regime is formally entered. When a condition for fast surface diffusion at the tracer source is introduced, the upper limit for Harrison type-A diffusion kinetics is pushed up slightly to $\Lambda=0.7$. It is clear that a tracer atom need not sample a large number of grains and grain boundaries in order for its effective diffusivity to be given by the Hart Equation (Eq. 1). In fact, this result implies that the diffusion length need only be roughly equal to the spacing between the (parallel) grain boundary slabs. This result is in fact similar to what was found for dislocation pipe diffusion where the upper limit for Harrison type-A diffusion kinetics is known from a near-exact analysis to occur at $\Lambda=0.1$ [4].

Divinski et al. [5] examined the transition from the Harrison Type-A kinetics regime to the Type-B kinetics regime more closely by focusing especially on the intermediate region. Similar to the findings of the earlier study by the present authors [3], they found that the concentration depth profiles (from a thin film tracer source) are well linear when plotted against the penetration depth (y) squared (i.e. Gaussian) for $\Lambda \leq 0.3$. At larger values of $\Lambda$ the profiles become curved and only a crude estimate of $D_{\text {eff }}$ is possible although this can be improved if only the first few points are used. They showed that the Harrison Type-B kinetics regime is probably not really well established until $\Lambda$ is approximately equal to 4 . Between $0.3 \leq \Lambda \leq 4$ they identified an intermediate region, formally called the 'AB transition regime', where the profiles show a pronounced negative curvature in a $y^{6 / 5}$ plot. Divinski and Larikov [6] had earlier provided an analysis in this region wherein the depth profiles can be plotted against $y^{3 / 2}$ and the grain boundary diffusion coefficient can be extracted provided $\delta$ is known or estimated:

$$
D_{g b} \approx \frac{16.65 D_{\ell}^{0.1}}{(1+\delta / d) \delta^{0.2} t^{0.9}}\left(-\frac{\partial \ln C}{\partial y^{3 / 2}}\right)^{-4 / 3}
$$

The results described above refer to the parallel grain boundary slab model where there is of course no closure of the grains. In a diffusion experiment, it can be expected that tracer atoms will traverse a number of grains in the diffusion time. Accordingly, it is important to determine whether the transition point changes as a result of this. In the present study, we use Monte Carlo simulation of tracer concentration depth profiles to explore the location of the transition from Harrison Type-A kinetics regime to the Type-B kinetics regime transition for a more realistic model in which the grains are cubic. Since there is now no exact analytical reference point (though the MaxwellGarnett Equation [7], $d=2,3$ for 2 and 3 dimensional models respectively:

$$
D_{e f f}=\frac{D_{g b}\left(d D_{\ell}+(d-1) g\left(D_{g b}-D_{\ell}\right)\right)}{g D_{l}+(d-g) D_{g b}}
$$

comes close [8]) we use an independent Monte Carlo calculation of the effective diffusivity by way of the Einstein Equation to provide the reference point.

\section{Method of Computation and Model}

The present study was performed by making use of the Lattice Monte Carlo method. Let us very briefly discuss the essence of this method. The lattice model on which it is based was originally conceived as a very simple model for describing the actual atomistics of grain boundary diffusion [9]. Later, the model was greatly broadened to deal with a wide range of multi-scale mass diffusion and thermal diffusion problems where the particles exploring the lattice and the lattice itself lose all atomistic significance $[10,11]$. The direction of the random walks of the particles and the evaluation of the spatially dependent jump frequencies (which represent the diffusivities) is made using 
random numbers. In the present study, the grain boundary is represented by a single plane of lattice sites in which the grain boundary diffusivity of the diffusant is associated with a single jump frequency $\left(w_{\mathrm{gb}}\right)$. The rest of the lattice represents the grains and has a jump frequency $\left(w_{1}\right)$ associated with the bulk diffusivity of the diffusant. When the jump frequencies to and from ( $w_{1 \rightarrow \mathrm{gb}}$ and $w_{\mathrm{gb} \rightarrow 1}$ respectively) the grain boundary are different from the other jump frequencies then segregation of the diffusant (quantitatively described by the segregation factor $s=w_{\mathrm{l} \rightarrow \mathrm{gb}} / w_{\mathrm{gb} \rightarrow \mathrm{l}}$ ) to the grain boundary can be formally described. In the present paper, however, we restrict ourselves to self-diffusion. To simulate a thin-film tracer source $10^{6}$ particles are created and released from the surface and allowed to diffuse for a time $t$ (proportional to the number of jump attempts per particle).

The principal outputs of the Lattice Monte Carlo calculations are the tracer concentration (depth) profiles for the grain boundary diffusion in exactly the same fashion as they appear in experimental tracer measurements. These concentration profiles are built up simply by determining the number of particles that have reached a given distance from the tracer source plane after time $t$.

In addition, the effective diffusivity $D_{\text {eff }}$ of the model material is also calculated simply by using the Einstein Equation in the long time limit (with the particles now created at random positions in the lattice (such positions would need to be weighted if there is segregation in the problem):

$$
D_{e f f}^{\text {Ein }}=\frac{<R^{2}>}{6 t}
$$

where $R$ is the vector displacement of a particle in time $t$.

We model the grain boundary tracer diffusion problem by constructing a $3 \mathrm{D}$ structure consisting of cubic grains each of equal volume. We build the structure in such a way that no four cubes have a common edge (see Fig. 1). The resulting structure has a 'geometric' anisotropy of the grains that can be seen by comparing two diffusion directions in the structure as shown in Figs. 1a and 1b. (It can be seen that the third principal direction (the $\mathrm{z}$-direction) is equivalent to the $\mathrm{x}$ - direction.)

For the grain boundary numerical diffusion profile calculated in a three dimensional manner it is usually found that the resulting profile is somewhat wavy and is impossible to be analyzed intelligently. To avoid this problem in our calculations we introduced an appropriate averaging procedure for the position of the source plane. This can be done, for example, by moving the source plane in the direction of the diffusion through the elementary distance systematically and then weighing the contribution from each obtained profile equally.

For diffusion from a thin-film tracer source the first section of the concentration depth profile is invariably approximately Gaussian. When the contribution to diffusion from the grain boundaries is relatively minor, this Gaussian region describes only the lattice diffusion coefficient $D_{1}$ of the diffusant. As the grain boundary contribution to diffusion grows, the diffusion coefficient measured in this Gaussian region increases until it finally becomes the effective diffusion coefficient $D_{\text {eff }}$ for diffusion of the diffusant in the material and the Harrison Type-A kinetics regime is formally entered. (Between these two kinetics regimes there is an apparent intermediate $A B$ region as mentioned above.)

In our study, we concentrated on the Harrison Type-A kinetics regime. In this regime, the penetration profiles from a thin-film source can be analyzed in the usual Gaussian form $(\ln \mathrm{c}) \mathrm{vs} \mathrm{y}^{2}$ :

$$
c=\exp \left(-\frac{y^{2}}{2 D_{\text {eff }} t}\right)
$$


We processed the first part of the concentration depth profile. This should give the effective diffusivity if Harrison Type-A kinetics is followed. Our target is to process systematically all the concentration profiles obtained in the numerical simulations and plot the resulting effective diffusivities relative to the actual effective diffusivities of the samples obtained by means of the Einstein Equation (Eq. 4) analysis.

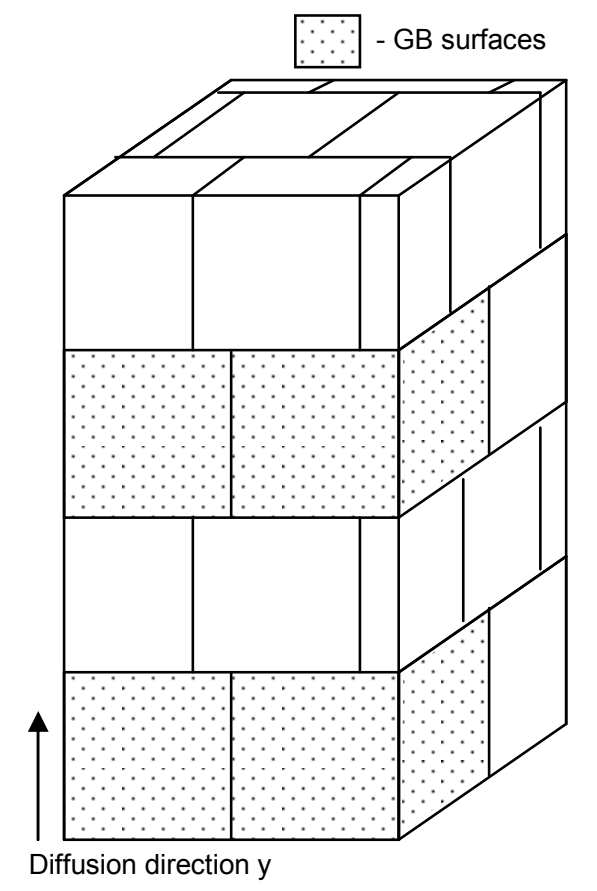

(a)

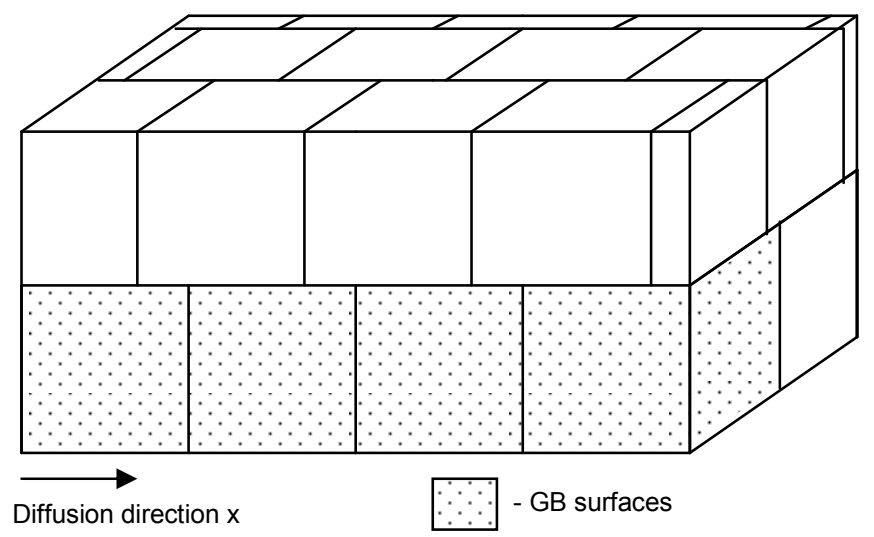

(b)

Figure 1. Schematic representation of the numerical models for the grain boundary diffusion problem: a) diffusion is in the $\mathrm{y}$ direction, the source plane is at $\mathrm{y}=0 ; \mathrm{b}$ ) diffusion is in the $\mathrm{x}$ direction, the source plane is at $\mathrm{x}=0$. 


\section{Results and Discussion}

In Fig. 2 we present results of a systematic analysis of the diffusion concentration profiles obtained in Lattice Monte Carlo simulations of the grain boundary diffusion processes (grains modelled by cubes). We see that the transition value of $\Lambda$ for this model is now very close to 3.5 .

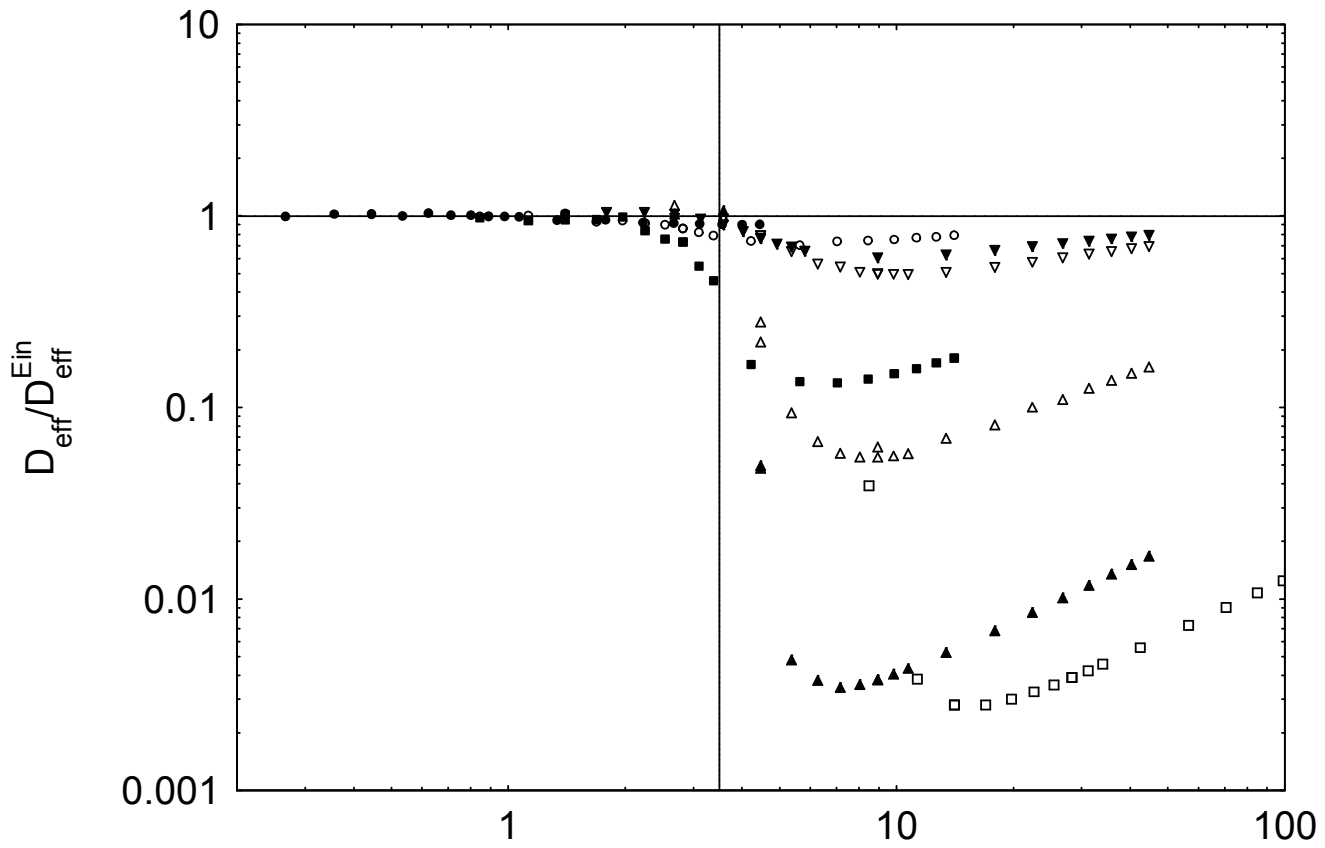

$\Lambda$

Figure 2. $D_{\text {eff }} / D_{\text {eff }}^{\text {Ein }}$ as a function of $\Lambda$ for various values of $\beta$ for the case of cubic grains (Figure $1 \mathrm{a}, \mathrm{b})$.

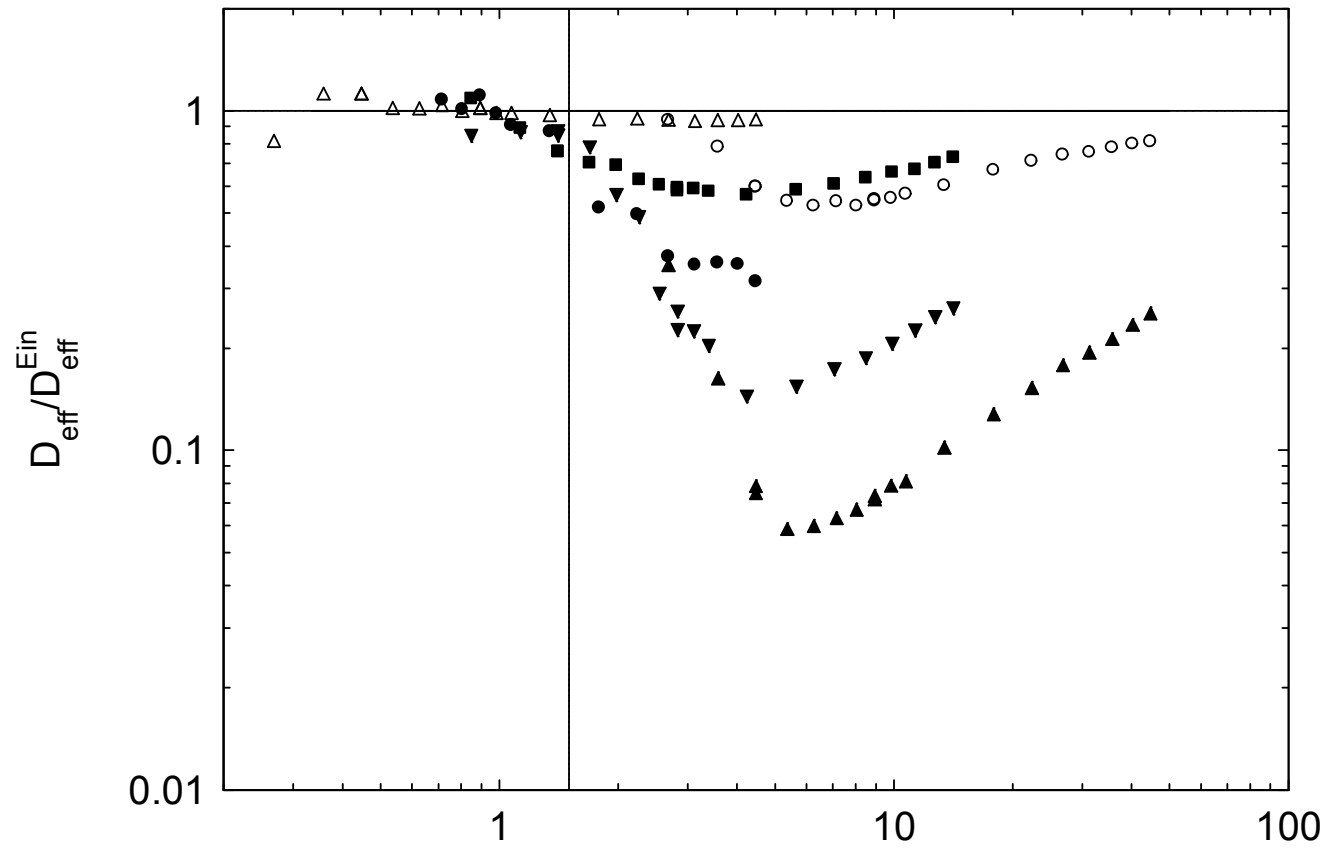

$\Lambda$

Figure 3. $D_{\text {eff }} / D_{\text {eff }}^{\text {Ein }}$ as a function of $\Lambda$ for various values of $\beta$ for the case of square grains (square planar arrangement). 
This is roughly an order of magnitude higher than in the case of the parallel grain boundary slab model. This finding raises a question: is this difference only due to the modeling of diffusion in closed grains or is the difference due to the change in the 'effective' dimensionality of the diffusion problem. To answer this question we performed numerical simulations for the two-dimensional model consisting of square grains. The result is shown in Fig. 3. Here we have plotted $D_{\text {eff }} / D_{\text {eff }}^{\text {Ein }}$ calculated for various values of $\Lambda$ for the case of squares arranged in a square planar fashion. We can see that critical value of $\Lambda$ for this model is about 1.5. Thus all three models for the grain boundary diffusion - parallel slabs, 2D - squares and 3D - cubes show different critical values of $\Lambda$ : these are $0.4,1.5$ and 3.5 respectively. Therefore dimensionality and geometric shape are both important factors in the parametric analysis of the grain boundary diffusion problem.

\section{Summary}

We modelled the grain boundary tracer diffusion problem by constructing a 3D structure consisting of cubic grains each of equal volume. We built the structure in such a way that no four cubes have a common edge. It was shown that the transition point between Harrison Type-A and Type-B kinetics regimes occurs at $\Lambda \approx 3.5$. This is roughly an order of magnitude higher than for the extensively studied case of parallel grain boundary slabs. For two-dimensional squares the transition point was shown to occur at $\Lambda \approx 1.5$. Thus we can draw the conclusion that dimensionality and geometric shape are both important factors in the parametric analysis of the grain boundary diffusion problem.

\section{Acknowledgements}

The Australian Research Council is acknowledged for its support of this work under the Discovery Projects Grants Scheme.

\section{References}

[1] L.G. Harrison: Trans. Farad. Soc. Vol. 57 (1961), p. 1191.

[2] E.W. Hart: Acta Metall. Vol. 5 (1957), p. 597.

[3] I.V. Belova and G.E. Murch: Phil. Mag. Vol. 81 (2001), p. 2447.

[4] A.D. Le Claire and A. Rabinovitch, in Diffusion in Crystalline Solids, edited by G.E. Murch and A.S. Nowick (Academic Press, Orlando 1984).

[5] S.V. Divinski, F. Hisker, Y.-S. Kang, J.-S. Lee and Chr. Herzig: Zeit. Metallk. Vol. 93 (2002), p. 256.

[6] S.V. Divinski and L.N. Larikov: Defect and Diffusion Forum Vol. 143-147 (1997), p. 1469.

[7] J.C. Maxwell: Phil. Trans. Roy. Soc. London Vol. 203 (1904), p. 385.

[8] I.V. Belova and G.E. Murch: J. Metastable Nanocryst. Matls. Vol. 19 (2004), p. 25.

[9] P. Benoist and G. Martin: Thin Solid Films Vol. 25 (1975), p. 181.

[10] I.V. Belova and G.E. Murch: Solid State Phenomena Vol. 129 (2007), p. 1.

[11] I.V. Belova and G.E. Murch, in Cellular and Porous Materials. Thermal Properties Simulation and Prediction, edited by A. Öchsner, G.E. Murch and J.S. de Lemos (Wiley - VCH; Weinheim) in press. 
Diffusion in Solids and Liquids III

doi:10.4028/www.scientific.net/DDF.273-276

Exploration of the Transition from Harrison Type-A Kinetics to Type-B Kinetics Regimes in Grain Boundary Diffusion

doi:10.4028/www.scientific.net/DDF.273-276.425 